\title{
Utility of the Addenbrooke's Cognitive Examination in Amyotrophic Lateral Sclerosis
}

\author{
Sneha Chenji, Dennell Mah, Wendy Johnston, Richard Camicioli, Nancy Fisher, \\ Sanjay Kalra
}

\begin{abstract}
Objective: Amyotrophic lateral sclerosis (ALS) is a neurodegenerative condition that primarily affects motor neurons. Cognitive changes are reported in $25 \%-50 \%$ of patients, secondary to frontotemporal involvement. The objective of this study was to evaluate the utility of a screening tool, the Addenbrooke's Cognitive Examination (ACE), in ALS patients. Methods: In this retrospective cross-sectional study, performance on the ACE was compared between 55 ALS patients and 49 healthy controls. The validation of the ACE in ALS patients was explored using a neuropsychometric battery. Correlations between the ACE and clinical variables such as the ALS Functional Rating Scale-Revised (ALSFRS-R) and forced vital capacity were computed. Results: A higher percentage of patients were below cut-off scores, although this remained non-significant between the patient and control groups. The ACE did not reveal significant differences between ALS patients and controls. The scores on the ACE displayed moderate correlations with our neuropsychometric battery for some domains, whereas others showed poor or no associations. Poor ACE Total was associated with lower ALSFRS-R and finger-tapping scores. Conclusions: Performance on the ACE was comparable between patients and controls. Associations with motor function pose a challenge to accurate interpretation of ACE performance. It is likely that patients with poor cognition have greater disability, or that poor ACE performance reflects reduced motor ability to perform the task. This raises concern for the utility of the ACE as a screening tool in ALS patients, especially since recent versions of the ACE continue to include motor-based tasks.
\end{abstract}

RÉSUMÉ: Utilité de l'épreuve cognitive d'Addenbrooke dans le cas de la sclérose latérale amyotrophique (SLA). Objectif: La sclérose latérale amyotrophique (SLA) constitue une affection neuro-dégénérative qui affecte principalement les motoneurones. Des changements d'ordre cognitif sont signalés chez environ 25 à $50 \%$ des patients et résultent d'une dégénérescence fronto-temporale. La présente étude a cherché à évaluer l'utilité d'un outil de dépistage, l'épreuve cognitive d'Addenbrooke, dans le cas de patients atteints de la SLA. Méthodes: Dans cette étude transversale rétrospective, on a comparé les résultats à l'épreuve cognitive d'Addenbrooke de 55 patients atteints de SLA à ceux de 49 patients en santé faisant partie d'un groupe témoin. Pour valider les résultats obtenus par les patients atteints de SLA, nous avons utilisé une batterie de tests neuro-psychométriques. On a ensuite calculé des corrélations entre les résultats à l'épreuve cognitive d'Addenbrooke et des variables cliniques obtenues au moyen de la ALS Functional Rating Scale Revised (ALSFRS-R) et d'un test de spirométrie, le Forced Vital Capacity (FVC). Résultats: Une proportion plus élevée de patients a obtenu des résultats sous les valeurs limites. À cet égard, la différence entre les résultats des patients atteints de SLA et ceux du groupe témoin s'est avérée non significative. Pour certains aspects, on a aussi pu établir une corrélation modérée entre les résultats à cette évaluation et ceux obtenus à la suite de notre batterie de tests neuro-psychométriques. Pour d'autres aspects, on n'a pu trouver qu'une faible association ou pas d'association du tout. Enfin, un faible résultat total à l'épreuve cognitive d'Addenbrooke a été associé à de plus faibles résultats à l'ALSFRS-R et au test de tapotement du doigt. Conclusions: Les résultats obtenus à l'épreuve cognitive d'Addenbrooke, qu'il s'agisse de patients atteints de SLA ou ceux du groupe témoin, se sont avérés comparables. Cela dit, les corrélations établies avec des fonctions motrices pose un problème si l'on veut interpréter correctement les résultats obtenus à l'épreuve cognitive d'Addenbrooke. Ainsi, il y a fort à parier que les patients aux prises avec une cognition déficiente souffrent d'une plus grande incapacité ou bien que de faibles résultats à l'épreuve cognitive d'Addenbrooke témoignent d'une capacité motrice réduite lorsqu'il est temps d'accomplir une tâche. Chez des patients atteints de la SLA, cela ne va pas sans susciter des doutes quant à l'utilité de l'épreuve cognitive d'Addenbrooke comme outil de dépistage, et ce, plus particulièrement dans la mesure où les versions récentes de cette épreuve continuent à inclure des tâches de nature motrice.

Keywords: Amyotrophic lateral sclerosis, Cognitive impairment, Addenbrooke's Cognitive Examination, Cognitive screening doi:10.1017/cjn.2018.68

Can J Neurol Sci. 2018; 45: 527-532

\section{INTRODUCTION}

Amyotrophic lateral sclerosis (ALS) is a neurodegenerative disorder involving the progressive loss of motor neurons. In addition, cognitive impairments with a frontotemporal profile have been reported in $25 \%-50 \%$ of ALS patients, and about
From the Neuroscience and Mental Health Institute, University of Alberta, Edmonton, Alberta, Canada (SC, SK); Division of Neurology, Department of Medicine, University of Alberta, Edmonton, Alberta, Canada (DM, WJ, RC, SK); Division of Neurosciences, Alberta Health Services, Edmonton, Alberta, Canada (NF).

Received August 28, 2017. Final Revisions Submitted May 2, 2018. Date of ACCEPTANCE MAY 14, 2018.

Correspondence to: S. Chenji, Neuroscience and Mental Health Institute, University of Alberta, 562 HMRC, 1131387 Ave NW, Edmonton, AB, Canada T6G 2S2.

Email: chenji@ualberta.ca 
5\%-10\% patients meet criteria for frontotemporal dementia (FTD). ${ }^{1}$ However, identifying these changes in patients with ALS in clinic settings remains challenging, as the changes can be subtle and variable, and tests probing frontotemporal cognitive domains can be time-consuming and require examiner expertise-factors that are difficult to accommodate in a busy clinic.

To bridge this gap, screening tools are increasingly being used in clinics, especially in cognitive clinics. ${ }^{2-5}$ Screening tools have an advantage of assessing various domains of cognitive function as opposed to single tests, and they are time-efficient in a busy clinic setting. The Addenbrooke's Cognitive Examination (ACE) was developed with the aim of distinguishing FTD from Alzheimer's dementia (AD, 3). It included six domains with a maximum score of 100 . The cut-off scores 88 and 83 have high sensitivity and specificity to cognitive impairment, respectively. ${ }^{3}$ In addition, a Verbal Fluency, Language, Orientation and Memory (VLOM) ratio ${ }^{\text {a }}$ distinguished AD and FTD; in participants with a VLOM ratio $\geq 3.2$, there is a $76 \%$ probability of $\mathrm{AD}$, whereas those with VLOM ratio $\leq 2.2$ have a $71 \%$ probability of FTD.

Considering the sensitivity of the ACE to frontotemporal changes, ${ }^{3}$ our retrospective cross-sectional study evaluated the utility of the ACE for ALS patients. Our aims were to (i) compare performance of patient and control groups on the ACE; (ii) evaluate the performance on the ACE against formal neuropsychometric tests; and (iii) identify associations between the ACE and clinical variables such as disability, respiratory function and finger tapping. We hypothesised that patients would perform poorly on the ACE as compared with controls. We hypothesised that the ACE would have construct validity as indicated by associations between scores on the ACE and the neuropsychometric tests. ${ }^{6}$ Finally, considering the motor components of some tasks on the ACE, we hypothesised that factors such as motor disability would be associated with poor ACE scores.

\section{MeTHODS}

\section{Sample}

The ACE data in the present study were collated from five studies initiated by the ALS Research Program between 2006 and 2008 at the University of Alberta. To ensure minimal bias during data collection, neuropsychometric training was provided by a single psychometrician for test administration and scoring. This training was completed by research assistants and students involved in the studies. The neuropsychometric protocol was kept consistent for participants within each study. All studies were approved by the Health Research Ethics Board, and a formal consent was obtained from all participants for the corresponding studies. The ACE was administered on 59 ALS patients meeting criteria for possible, probable or definite $\mathrm{ALS} ;{ }^{7}$ five were unable to complete the ACE owing to motor deficits and were excluded from further analysis. Our final sample included 54 patients (Table 1). Patients had moderate to low disease burden as reflected by their ALS Functional Rating Scale-Revised (ALSFRS-R ${ }^{8}$ ) and forced vital capacity (FVC). In total, 47 healthy volunteers without a history of neurological or psychiatric conditions were included (Table 1). Differences in education were accounted for in the statistical model used for analysis.

\section{Instrument}

The ACE evaluates six aspects of cognition: orientation, attention, memory, verbal fluency, language and visuospatial ability, and incorporates items from the Mini-Mental State Examination (MMSE). ${ }^{2,3}$ The total administration time is approximately 15-20 minutes.

\section{Neuropsychometric Tests}

A subset of 35 ALS patients and 41 healthy controls were administered additional neuropsychometric tests assessing additional cognitive domains such as executive functions, language, learning and memory, and visuospatial abilities. In total, 23 patients and 17 controls completed digit span (forward and backward) from Wechsler Adult Intelligence Scale-Revised (WAIS-R), ${ }^{9}$ digit ordering test, ${ }^{10}$ Boston Naming Test $(\mathrm{BNT})^{11}$ and Benton Judgement of Line Orientation. ${ }^{12}$ In total, 21 participants also completed the Stroop Test, ${ }^{13,14}$ and a smaller sub-group completed Wechsler's Test of Adult Reading ${ }^{15}$ ) and the California Verbal Learning Test-II (CVLT-II ${ }^{16}$ ). In total, 12 patients and 24 controls were administered only verbal fluency (letter $\mathrm{F}$ and $\mathrm{A}^{17,18}$ ). Mood was assessed using the Beck's Depression Inventory-II (BDI-II) in 19 patients and 17 controls (see Supplementary Table 4).

\section{Statistical Analysis}

IBM SPSS software package (version 21) was used for analysis. ${ }^{19}$ Normality was tested for the ACE and neuropsychometric

Table 1: Participant demographics

\begin{tabular}{|c|c|c|c|}
\hline Demographics & Patients $(n=54)$ & Controls $(n=49)$ & $p$ \\
\hline Age (years) & $59.7 \pm 10.7$ & $56.6 \pm 11.0$ & n.s. \\
\hline Education (years) & $13.4 \pm 2.8$ & $15.8 \pm 3.4$ & $<0.01$ \\
\hline Gender (M:F) & $41: 13$ & $16: 31$ & 0.001 \\
\hline Onset (Limb:Bulbar)* & $35: 17$ & - & - \\
\hline Symptom duration (months, mean $\pm \mathrm{SD}$, median [range]) & $23 \pm 24(13.5,5-110)$ & - & - \\
\hline FVC (\% reference) & $89.2 \pm 17.1$ & - & - \\
\hline ALSFRS-R & $40.0 \pm 5.3$ & - & - \\
\hline
\end{tabular}

ALSFRS-R = ALS Functional Rating Scale-Revised; FVC = forced vital capacity.

*Available in 52 patients. 


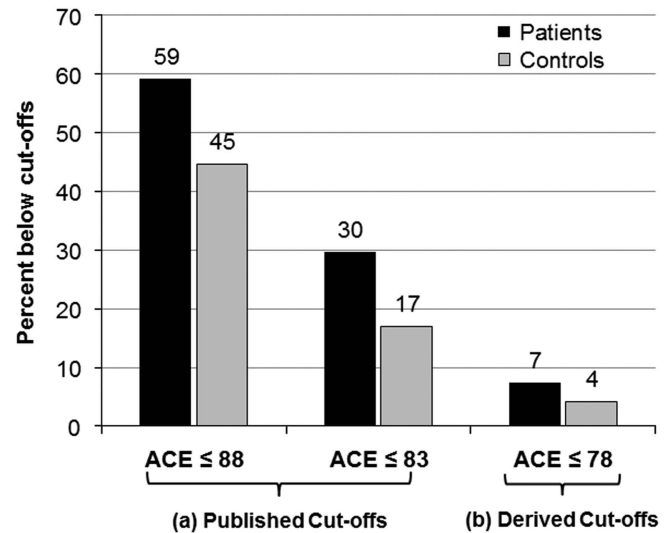

Figure 1: Percentage of patients and controls below cut-off scores on the Addenbrooke's Cognitive Examination (ACE). There were no significant differences in the distribution of patients and controls below (A) the published cut-off scores of $A C E \leq 88$ for high sensitivity $\left(\chi^{2}=2.7, p=0.09\right)$ or ACE $\leq 83$ for high specificity $\left(\chi^{2}=2.5, p=0.11\right)$, and $(B)$ the derived cut-off score ACE $\leq 78\left(\chi^{2}=0.5, p=0.47\right)$.

tests using either Shapiro-Wilk test or Kolmogorov-Smirnov tests (Supplementary Tables 1 and 2). Analysis of covariance with education as a covariate was used to compare group differences in performance on the ACE. The percentage of patients and controls below (a) published cut-off scores and (b) derived cut-off scores (5th percentile of the control group) were calculated and the distribution of participants was compared using Pearson's $\chi^{2}$ test. Spearman's correlations were calculated between the ACE and neuropsychometric tests for each group of participants. Associations between the ACE and clinical variables were also computed for patients. Statistical significance was accepted at $p<0.05$ and corrections for multiple comparisons (false discovery rate, FDR) were performed where applicable.

\section{RESUlTS}

\section{Group Comparisons}

The patient and control groups were matched for age $(p>0.05)$. However, they differed significantly for education and gender distribution (Table 1). There were no significant differences in performance on the ACE based on gender; thus, only education was used as a covariate in further group comparisons. There were no significant differences in performance on the total ACE score individual domains between patients and controls, except for a strong trend in the visuospatial ability of the ACE (Supplementary Table 3). Neuropsychometric tests indicated a significant difference for BDI-II $(p<0.01$, FDR-corrected). At more lenient thresholds, digit ordering test ( $p=0.05$, uncorrected) and Stroop non-interference condition (words, $p<0.01$ uncorrected) were significantly lower as compared with controls. Judgement of line orientation was lower in controls as compared with patients $(p=0.04$, uncorrected).

\section{Participants Below Cut-Off Scores}

A higher percentage of patients were below cut-off scores as compared with controls, although this was not statistically significant (Figure 1). The published cut-off scores were proposed by ${ }^{\mathrm{a}}$ VLOM Ratio $=($ Verbal Fluency + Language $) /($ Orientation + Memory $)$
Mathuranath et al, ${ }^{3}$ whereas our derived cut-off scores are the 5 th percentiles of our control group.

\section{ACE and the Neuropsychometric Battery}

Spearman's correlations of the ACE and performance on neuropsychometric tests for each participant group indicated moderate-to-strong positive associations of ACE Total Score memory and verbal fluency domains with verbal fluency (letter F and A; Supplementary Table 5). Higher ACE Total indicated a significant association with lower language (BNT) performance in controls $(p<0.05$, uncorrected), but not patients. A significant association with higher ACE Total, memory and CVLT shortdelay recall was noted in patients, but not controls $(p<0.05$, uncorrected). Language scores on the ACE did not show any associations with Boston Naming Test for patients or controls. Visuospatial abilities did not reveal any significant associations either. Higher VLOM ratios were associated with lower performance on CVLT short-delay recall for controls $(p<0.05$, uncorrected), but not patients. CVLT long-delay recall showed trends with ACE Total, MMSE and VLOM ratios in controls $(p<0.09)$ and only with VLOM ratio in patients $(p<0.09)$. Non-meaningful associations were noted between ACE orientation, judgement of line orientation and ACE memory and Stroop interference performance in controls.

\section{ACE and Clinical Variables}

Spearman's correlations with clinical variables indicated that lower ALSFRS-R was moderately associated with lower ACE Total Score (Spearman's $\rho=0.6, p<0.01$; Figure 2A) and lower memory score (Spearman's $\rho=0.5, p<0.05$ ). A significant association was also noted between finger-tapping and ACE Total performance (Spearman's $\rho=0.5, p<0.05$; Figure 2B). There was a trend towards associations between lower ALSFRS-R and poor ACE Verbal Fluency score (Spearman's $\rho=0.4, p=0.07$; Supplementary Figure). There were no significant correlations between performance on the ACE and FVC.

\section{DiscuSSION}

Our study evaluated the utility of the ACE as a screening tool for ALS. We investigated this by (i) comparing performances of ALS patients and healthy controls, (ii) validating the ACE against a standard neuropsychometric battery and (iii) associating performance on the ACE with clinical variables such as the ALSFRS-R.

\section{Group Comparisons and Per cent Below Cut-Off Scores}

We report no significant differences in performance between patients and controls on the ACE. A strong trend was noted in visuospatial ability, with controls performing lower than patients; however, this was driven by 1 outlier. The trend was eliminated when the control was excluded from analysis. Although a higher proportion of patients scored below published and derived cut-off scores on the ACE, there were no significant differences in the distribution of participants below (a) published and (b) derived cut-off scores. To the best of our knowledge, this is the only report of the use of the original ACE in ALS. We acknowledge that the ACE is an older test while recent revised versions have been used in the literature. Studies have emerged reporting the application of the revised version of the ACE (ACE-R) ${ }^{20}$ in ALS to either identify cognitive impairment in 
(a)

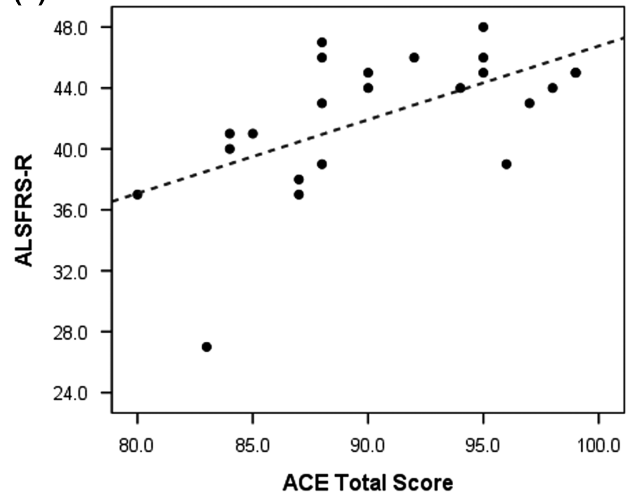

(b)

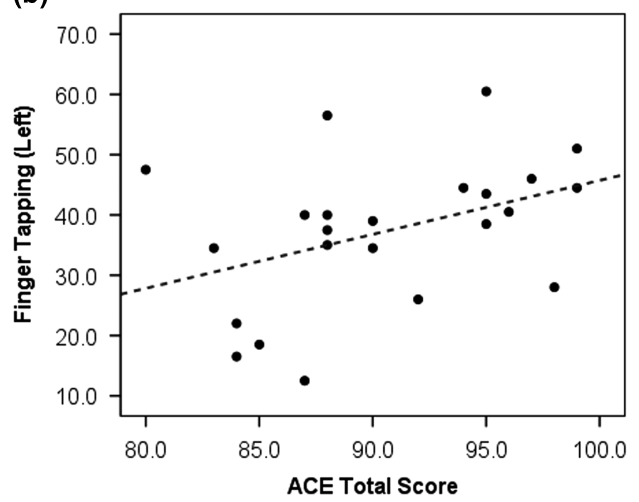

Figure 2: Scatterplot graphs indicating linear associations between (A) amyotrophic lateral sclerosis Functional Rating Scale-Revised (ALSFRS-R) and Addenbrooke's Cognitive Examination (ACE) Total Score (Spearman's $\rho=0.6, p<0.01$ ), and (B) Finger-tapping and ACE Total score (Spearman's $\rho=0.5$, $p<0.05)$.

the ALS-FTD spectrum ${ }^{21-25}$ or as a screening tool for recruitment. ${ }^{26}$ The ACE-R incorporates content changes to the domains of memory, language and visuospatial abilities of the ACE to ensure a more distributed scoring across the domains ${ }^{20}$ and improved diagnostic accuracy. ${ }^{27}$ Our recruitment began before the publication of the ACE-R, when there were limited cognitive screening tools and no specific cognitive tests for ALS. The ACE provided the option for frontotemporal assessment, much needed for evaluating the frontotemporal lobar degeneration in ALS. We continued using the original ACE to maintain consistency between studies.

Across the ALS-FTD spectrum, there are inconsistent reports of impairment using the ACE-R. One study reported comparable performance on the ACE-R between ALS and behavioural variant FTD patients; both groups performed poorly as compared with controls. $^{21}$ The study included 5 of 20 ALS patients meeting criteria for FTD, which explains the comparable performance between the patient groups given the small sample sizes. In contrast, another study reported poor ACE-R scores in ALS-FTD patients, and comparable scores between non-demented ALS patients and healthy controls. ${ }^{22}$ Using the ACE-R semantic deficits have also been reported in ALS patients with a graded performance as compared with other groups; order of language score from highest to lowest is controls, followed by ALS, ALS-FTD and semantic dementia patients. ${ }^{23}$ Higher age, female gender and lower education have been reported as potential determinants of cognitive impairments in ALS based on ACE-R scores. ${ }^{24}$

A recent study by $\mathrm{Hsieh}_{\mathrm{e}} \mathrm{al}^{28}$ utilised the mini Addenbrooke's Cognitive Examination version-III (M-ACE). The M-ACE included items assessing orientation in time, executive functions (animal fluency), visuospatial abilities (clock drawing) and learning and recall of an address. ${ }^{29}$ It was used in combination with Motor Neuron Disease Behavioural Scale (MiND-B) ${ }^{30}$ to determine cognitive and behavioural changes in the ALS-FTD spectrum. The authors reported that $90 \%$ of ALS-FTD patients and only $20 \%$ of non-demented ALS patients scored below cut-offs for the M-ACE, whereas the MiND-B questionnaire distinguished ALS patients with behavioural or cognitive impairments from non-demented ALS. Although recent versions of the ACE may have provided an advantage in identifying cognitive impairments, the studies include a wide range of cognitive profiles in ALS patients, with higher prevalence of ALS-FTD patients. In addition, language and memory domains contribute to $50 \%$ of the memory score in ACE$\mathrm{R}$ and ACE-III. These two domains include motor-based tasks that would require careful implementation of corrections for motor impairments. Although this is not a direct comparison, it remains a cautionary note given the construct of the ACE.

\section{ACE Scores and the Neuropsychometric Battery}

Higher scores on the ACE Total, memory and verbal fluency domains were associated with corresponding tests on the neuropsychometric battery, suggesting construct validity of these ACE domains. It was surprising to see no association between the language scores on the ACE and the Boston Naming Test or between visuospatial abilities and Judgement of Line Orientation. One possibility is that these tests were performed on a small subset of participants and hence may lack sufficient power in our analysis. Additional details outlining the associations are included in Supplementary Table 5.

\section{ACE Scores and Clinical Variables}

Poor ACE scores for verbal fluency were associated with lower ALSFRS-R (greater disability), indicating that patients with greater motor dysfunctions perform poorly on the ACE. One possibility is that patients with motor dysfunction indeed had cognitive impairments. ${ }^{31}$ Alternatively, we could consider poor performance on the ACE to be associated with reduced motor ability to perform the task, and not associated with cognitive impairment. This raises concern regarding the ability of the ACE to identify cognitive impairment independent of motor dysfunctions. ${ }^{32}$ Anecdotal reports by Abrahams et $\mathrm{al}^{33}$ indicated that only $59 \%$ of ALS patients completed all the domains of the ACE-R. The domains that were completed differed among patients owing to their varying range of disabilities and thereby restricted comparisons.

More recent versions of the ACE have been published since our last data collection. ACE-III and M-ACE are reported to be more sensitive in screening for cognitive impairments in clinical populations. ${ }^{34,35}$ These versions still require modifications to account for motor disability and do not address social cognition and behavioural changes in ALS. Studies also report utility of ALS-specific tools 
such as the ALS Cognitive Behavioural Screen ${ }^{36}$ and Edinburgh Cognitive and Behavioural ALS Screen (ECAS) ${ }^{37}$ and would be of interest for consideration in ALS clinics. A recent study compared performance of ALS patients and neuromuscular disease controls on cognitive screening tools (ACE-III, Frontal Assessment Battery [FAB] and ECAS executive domain). The authors report significantly lower performance on total scores of the ACE-III and the FAB for ALS patients. ${ }^{38}$ Impairment was identified in $30 \%$ of ALS patients $(n=81)$ on the ACE-III, $14 \%$ on the FAB and $22 \%$ on the ECAS executive domain $(n=41)$. Motor impairments in that study were corrected for by converting scores into per cent correct responses. However, the authors do not reveal whether patients impaired on ACE-III were also impaired on the FAB or ECAS executive domain.

In conclusion, we report non-significant differences in performance on the ACE in patients compared with controls. Although some of the ACE domains were positively associated with neuropsychometric battery, indicating construct validity, other domains such as language and visuospatial abilities showed poor or no associations with the neuropsychometric battery. The association between motor disability and poor ACE scores raises concern for its clinical utility in ALS. Although recent versions of the ACE such as the M-ACE have been explored in combination with behavioural screens, its utility in ALS clinics where patients show a varying range of motor dysfunctions needs further validation. We did not assess behaviour in our sample, whereas mood was assessed for a smaller subset; evaluation of these domains should be considered for validating other ALS-specific tools.

\section{ACKNOWLEDGEMENTS}

The authors sincerely thank patients and controls for taking time and participating in our studies.

\section{Disclosures}

All authors have no conflicts of interest to disclose.

\section{Statement of Authorship}

SC was involved with collating ACE data, designing experiments for data analysis, interpretation and writing the manuscript. DM was involved in data collection and collating ACE data. WJ provided expert advice on data analysis, interpretation and editing the manuscript. RC and NF were involved in designing the core neuropsychometric battery in our studies. SK, the principal investigator, was involved in designing the experiments, provided advice on data analysis and editing the manuscript.

\section{SUPPLEMENTARY MATERIAL}

To view supplementary material for this article, please visit https://doi.org/10.1017/cjn.2018.68

\section{REFERENCES}

1. Phukan J, Elamin M, Bede P, et al. The syndrome of cognitive impairment in amyotrophic lateral sclerosis: a populationbased study. J Neurol Psychiatry. 2012;83(1):102-8.

2. Folstein MF, Folstein SE, McHugh PR. "Mini-mental state". A practical method for grading the cognitive state of patients for the clinician. J Psychiatr Res. 1975;12(3):189-98.

3. Mathuranath PS, Nestor PJ, Berrios GE, Rakowicz W, Hodges JR. A brief cognitive test battery to differentiate Alzheimer's disease and frontotemporal dementia. Neurology. 2000;55(11):1613-20.
4. Dubois B, Slachevsky A, Litvan I, Pillon B. The FAB: a frontal assessment battery at bedside. Neurology. 2000;55(11):1621-6.

5. Nasreddine ZS, Phillips NA, Bedirian V, et al. The Montreal Cognitive Assessment, MoCA: a brief screening tool for mild cognitive impairment. J Am Geriatr Soc. 2005;53(4):695-9.

6. Galton CJ, Erzinclioglu S, Sahakian BJ, Antoun N, Hodges JR. A comparison of the Addenbrooke's Cognitive Examination (ACE), conventional neuropsychological assessment, and simple MRI-based medial temporal lobe evaluation in the early diagnosis of Alzheimer's disease. Cogn Behav Neurol. 2005;18 (3): 144-50.

7. Brooks BR, Miller RG, Swash M, Munsat TL, World Federation of Neurology Research Group on Motor Neuron D. El Escorial revisited: revised criteria for the diagnosis of amyotrophic lateral sclerosis. Amyotroph Lateral Scler Other Motor Neuron Disord. 2000;1(5):293-9.

8. Cedarbaum JM, Stambler N, Malta E, et al. The ALSFRS-R: a revised ALS functional rating scale that incorporates assessments of respiratory function. BDNF ALS Study Group (Phase III). J Neurol Sci. 1999;169(1-2):13-21.

9. Wechsler D. WAIS-R: Wechsler adult intelligence scale-revised. New York, NY: Psychological Corporation; 1981.

10. Werheid K, Hoppe C, Thone A, Muller U, Mungersdorf M, von Cramon DY. The Adaptive Digit Ordering Test: clinical application, reliability, and validity of a verbal working memory test. Arch Clin Neuropsychol. 2002;17(6):547-65.

11. Mack WJ, Freed DM, Williams BW, Henderson VW. Boston Naming Test: shortened versions for use in Alzheimer's disease. J Gerontol. 1992;47(3):P154-8.

12. Benton AL, Varney NR, Hamsher KD. Visuospatial judgment. A clinical test. Arch Neurol. 1978;35(6):364-7.

13. Stroop JR. Studies of interference in serial verbal reactions. J Exp Psychol. 1935;18(6):643-62.

14. Golden CJ. Identification of brain disorders by the Stroop Color and Word Test. J Clin Psychol. 1976;32(3):654-8.

15. Wechsler D. Wechsler Test of Adult Reading: WTAR. San Antonio, TX: Psychological Corporation; 2001.

16. Delis DC, Kramer JH, Kaplan E. CVLT-II: California Verbal Learning Test: Adult version. Manual. San Antonio, TX: Psychological Corporation; 2000.

17. Borkowski JG, Benton AL, Spreen O. Word fluency and brain damage. Neuropsychologia. 1967;5(2):135-40.

18. Spreen O, Benton AL. Neurosensory center comprehensive examination for aphasia. Victoria, BC: Neuropsychology Laboratory, Department of Psychology, University of Victoria; 1977.

19. Corp. I. IBM SPSS statistics for Windows, Version 21.0. Armonk, NY: IBM Corp; 2012.

20. Mioshi E, Dawson K, Mitchell J, Arnold R, Hodges JR. The Addenbrooke's Cognitive Examination Revised (ACE-R): a brief cognitive test battery for dementia screening. Int J Geriatr Psychiatry. 2006;21(11):1078-85.

21. Lillo P, Savage S, Mioshi E, Kiernan MC, Hodges JR. Amyotrophic lateral sclerosis and frontotemporal dementia: a behavioural and cognitive continuum. Amyotroph Lateral Scler. 2012;13(1): $102-9$

22. Savage SA, Lillo P, Kumfor F, Kiernan MC, Piguet O, Hodges JR. Emotion processing deficits distinguish pure amyotrophic lateral sclerosis from frontotemporal dementia. Amyotroph Lateral Scler Frontotemporal Degener. 2014;15(1-2):39-46.

23. Leslie FV, Hsieh S, Caga J, et al. Semantic deficits in amyotrophic lateral sclerosis. Amyotroph Lateral Scler Frontotemporal Degener. 2015;16(1-2):46-53.

24. Wei Q, Chen X, Zheng Z, et al. Screening for cognitive impairment in a Chinese ALS population. Amyotroph Lateral Scler Frontotemporal Degener. 2015;16(1-2):40-5.

25. De Silva D, Hsieh S, Caga J, et al. Motor function and behaviour across the ALS-FTD spectrum. Acta Neurol Scand. 2016;133 (5):367-72.

26. Staios M, Fisher F, Lindell AK, Ong B, Howe J, Reardon K. Exploring sarcasm detection in amyotrophic lateral sclerosis using ecologically valid measures. Front Hum Neurosci. 2013;7:178.

27. Larner AJ, Mitchell AJ. A meta-analysis of the accuracy of the Addenbrooke's Cognitive Examination (ACE) and the 
Addenbrooke's Cognitive Examination-Revised (ACE-R) in the detection of dementia. Int Psychogeriatr. 2014;26(4): 555-63.

28. Hsieh S, Caga J, Leslie FV, et al. Cognitive and Behavioral Symptoms in ALSFTD: detection, differentiation, and progression. J Geriatr Psychiatry Neurol. 2016;29(1):3-10.

29. Hsieh S, McGrory S, Leslie F, et al. The Mini-Addenbrooke's Cognitive Examination: a new assessment tool for dementia. Dement Geriatr Cogn Disord. 2015;39(1-2):1-11.

30. Mioshi E, Hsieh S, Caga J, et al. A novel tool to detect behavioural symptoms in ALS. Amyotr Lateral Scler Frontotemporal Degener. 2014;15(3-4):298-304.

31. Bak TH, Chandran S. What wires together dies together: verbs, actions and neurodegeneration in motor neuron disease. Cortex. 2012;48(7):936-44.

32. Abrahams S, Leigh PN, Harvey A, Vythelingum GN, Grise D, Goldstein LH. Verbal fluency and executive dysfunction in amyotrophic lateral sclerosis (ALS). Neuropsychologia. 2000;38 (6):734-47.
33. Abrahams S. ALS, cognition and the clinic. Amyotr Lateral Scler Frontotemporal Degener. 2013;14(1):3-5.

34. Hsieh S, Schubert S, Hoon C, Mioshi E, Hodges JR. Validation of the Addenbrooke's Cognitive Examination III in frontotemporal dementia and Alzheimer's disease. Dement Geriatr Cogn Disord. 2013;36(3-4):242-50.

35. Larner AJ. Mini-Addenbrooke's Cognitive Examination: a pragmatic diagnostic accuracy study. Int J Geriatr Psychiatry. 2015;30 (5):547-8.

36. Woolley SC, York MK, Moore DH, et al. Detecting frontotemporal dysfunction in ALS: utility of the ALS Cognitive Behavioral Screen (ALS-CBS). Amyotr Lateral Scler. 2010;11(3):303-11.

37. Abrahams S, Newton J, Niven E, Foley J, Bak TH. Screening for cognition and behaviour changes in ALS. Amyotr Lateral Scler Frontotemporal Degener. 2014;15(1-2):9-14.

38. Xu Z, Alruwaili ARS, Henderson RD, McCombe PA. Screening for cognitive and behavioural impairment in amyotrophic lateral sclerosis: frequency of abnormality and effect on survival. J Neurol Sci. 2017;376:16-23. 